\title{
The distribution of Clostridium difficile in the environment of South Wales
}

\author{
N. AL SAIF and J. S. BRAZIER* \\ Department of Medical Microbiology and Public Health Laboratory and *PHLS Anaerobe Reference Unit, \\ University Hospital of Wales, Heath Park, Cardiff CF4 4XW
}

\begin{abstract}
A large study of the distribution of Clostridium difficile in the environment of the Cardiff area of South Wales was performed with a methodology designed to maximise recovery. A total of 2580 samples was taken, with $184(7.1 \%)$ yielding isolates. The highest yield for $C$. difficile was obtained from river waters, with $14(87.5 \%)$ of 16 samples from four rivers positive, and from sea water samples with $7(44 \%)$ of 15 positive from six beaches on the Bristol Channel. In addition, 7 (46.7\%) of 15 samples of lake water were also positive. Twenty-two (21\%) of 104 soil samples, taken from random sites in Cardiff, were positive, as were $20 \%$ of environmental samples from four Cardiff hospitals. $C$. difficile was also isolated from $50 \%$ of eight swimming pool waters examined and $1(5.5 \%)$ of 18 of mains tap water. Carriage of $C$. difficile in 524 faecal samples of assorted farm animals was c. $1 \%$, and was $10 \%$ in dogs and $2 \%$ in cats. In private residences, the organism was present in $12(2.2 \%)$ of 550 samples. While $2.4 \%$ of 300 raw vegetable samples were positive, none of 107 assorted fish gut contents was. These findings indicate that $C$. difficile may be more widely distributed in the general environment, particularly water, than was previously thought.
\end{abstract}

\section{Introduction}

Clostridium difficile is a nosocomial pathogen responsible for many cases of antibiotic-associated diarrhoea (AAD) and pseudomembranous colitis $[1,2]$. Several studies have revealed the widespread presence of the organism in hospital wards and on the hands of nursing personnel [3-5], and infection is generally believed to be acquired nosocomially. Fewer studies have been directed at the environment outside hospitals and reports of $C$. difficile in the environment suggest either that its geographical distribution may be patchy, or that the different methodologies used were responsible for different isolation rates. For example, Hafiz from Sheffield, England [6] reported the organism in soil, sand and mud, and other workers in Korea [7] and Poland [8] have also found it in soil. However, one study in Perth, Western Australia [9], and a study of 20 random soil samples from Michigan, USA [4] both gave negative results.

Little is known about the prevalence of $C$. difficile in the domestic environment. Kim et al. [4] reported positive cultures from $12.2 \%$ of samples from floors and random surfaces in several rooms within a house occupied by a case of $C$. difficile diarrhoea. As a

Received 3 Aug. 1995; accepted 12 Jan. 1996.

Corresponding author: Dr J. S. Brazier. control, the home of a healthy person was similarly sampled and no positive cultures were reported.

The total number of potential animal reservoirs of $C$. difficile is large [9] but no studies have proved that they are the source of infection in man. Riley et al. [10] showed that $C$. difficile was the most commonly isolated organism in a survey of patients with diarrhoea presenting to general practices in Western Australia. These findings suggested exposure to the organism in the community.

The distribution of $C$. difficile in the general environment of the UK is largely unknown. A large study of the distribution of $C$. difficile in the environment of the Cardiff area of South Wales is described. The aim was to demonstrate how healthy individuals in the community may be exposed to $C$. difficile and to promote an understanding of the ecology of this organism.

\section{Materials and methods}

\section{Cultural methodology}

A commercially-prepared basal medium for the isolation of $C$. difficile (Brazier's Medium, Lab M 160-A; Lab M, Bury) [11], containing a selective supplement of cefoxitin $8 \mathrm{mg} / \mathrm{L}$ and cycloserine $250 \mathrm{mg} / \mathrm{L}$, was used. This medium incorporates the sodium salt of cholic acid to enhance the germination of spores; 
prolonged incubation of samples on this medium was required to maximise the recovery of $C$. difficile from the environment. The medium was prepared according to the manufacturer's instructions and poured in either $5.5-\mathrm{cm}$ contact plates (Bibby-Sterilin, Stone) for sampling directly from surfaces, or in $9-\mathrm{cm}$ petri dishes for routine culture. All incubations were performed in an anaerobic chamber with an atmosphere of $\mathrm{H}_{2} 10 \%, \mathrm{CO}_{2} 10 \%, \mathrm{~N}_{2} 80 \%$ at $37^{\circ} \mathrm{C}$. Cultures were incubated initially for $48 \mathrm{~h}$ and, if negative at this time, were re-incubated for up to a further $72 \mathrm{~h}$. C. difficile was recognised by screening typical colonies under long-wave ultra-violet light for characteristic yellowgreen fluorescence. These colonies were subcultured on non-selective Fastidious Anaerobe Agar (FAA) (Lab 90; Lab M) supplemented with horse blood $6 \%$ and incubated overnight. Confirmation of identification of C. difficile was by agglutination of colonies with the Microscreen Clostridium difficile latex reagent (Microgen Bioproducts Ltd, Camberley) and noting the characteristic odour of cultures.

\section{Testing isolates for toxin A production}

Environmental isolates of $C$. difficile were tested for their ability to produce toxin A (enterotoxin) by an enzyme immunoassay kit (TOX-A TEST, TechLab, Blacksburg, VA, USA). Isolates were inoculated on FAA supplemented with horse blood $6 \%$ and incubated anaerobically for $48 \mathrm{~h}$. A heavy suspension of the growth of each isolate was made in the diluent buffer provided with the kit. The assay instructions were then followed as if performing the test with a faecal suspension. Positive and negative controls were included in each batch.

\section{Water samples}

Samples of untreated water from rivers, lakes, drainage channels and the sea, in a 20 -mile radius of Cardiff, South Wales, were collected. Treated water samples from swimming pools and tap water from domestic supplies were also collected. Each sample was collected in $500-\mathrm{ml}$ sterile bottles with collection techniques recommended for standard water microbiological examination. Cultures for $C$. difficile were made by the membrane filtration method, passing $100-\mathrm{ml}$ volumes of each water sample through a $0.45-\mu \mathrm{m}$ pore size cellulose membrane filter (Millipore, Bedford). Membranes were placed on Brazier's selective agar, incubated anaerobically for up to 5 days, and the number of colonies was recorded as $\mathrm{cfu} / 100-\mathrm{ml}$. For the detection of organisms present in lower numbers, enrichment cultures were performed on all samples by placing three membranes of $100-\mathrm{ml}$ filtrate into cooked meat broth containing the selective agents. Each selective broth was incubated for $48 \mathrm{~h}$ before subculture on to selective agar. Direct cultures that were negative after $48 \mathrm{~h}$ were incubated for a further $72 \mathrm{~h}$ before being discarded.

\section{Faeces of dogs and cats}

Stool samples from 100 cats and 100 dogs were collected from two veterinary clinics and an animal shelter. Faeces were collected on a swab and placed immediately in charcoal transport medium. In the laboratory an equal volume of stool sample was added to absolute alcohol [12], mixed and left to stand for a minimum of $30 \mathrm{~min}$. The deposit was inoculated on selective agar and plates were incubated anaerobically for $48 \mathrm{~h}$. Any plates negative at $48 \mathrm{~h}$ were re-incubated for a further 3 days before being discarded.

\section{Faeces of assorted farm animals and fish gut contents}

Animals from a cattle and sheep market and 45 farms were sampled over a 7-month period. In total, 524 faecal samples were taken from 104 cattle, 100 sheep, 100 horses, 100 pigs and 120 mixed poultry. Swabs of the gut contents of 107 assorted sea and fresh water fish were taken at a fishery store. Culture for $C$. difficile was performed as described above for domestic animals.

\section{Soil samples}

Soil samples (104) from public parks, gardens, playgrounds and fields from sites in the Cardiff area were collected in sterile universal containers; $5 \mathrm{~g}$ of each sample were treated by alcohol shock [12] and c. $0.5 \mathrm{~g}$ of deposit was inoculated on the selective medium.

\section{Environmental surface samples in hospitals, veterinary clinics, nursing homes and private residences}

The distribution of $C$. difficile in hospitals, veterinary clinics, nursing homes and private residences was examined either by the use of direct contact plate cultures or moist swabs. Surfaces sampled included floors, tables, work surfaces, chairs, toilets, commodes, baths, carpets, windows, curtains and collections of dust. Swabs were plated out on selective medium and contact plates of $23.7 \mathrm{~cm}^{2}$ in area were applied to the surfaces under test.

\section{Raw vegetable samples}

A selection of 300 raw vegetables on sale in retail premises in Cardiff were cultured for $C$. difficile. The unwashed surfaces of the vegetables were sampled by contact plates. Vegetables included in the survey were cucumber, onion, tomato, potato, mushroom, cabbage, lettuce, carrot and radish.

\section{Results}

A total of 2580 samples was taken yielding 184 isolates 
and an overall recovery rate of $7.1 \%$ (Table 1 ). Toxin A was produced by $76 \%$ of the isolates.

\section{Water samples}

Fig. 1 shows the sampling points on the four rivers and the coastline of the Bristol Channel. There was a high yield from the four rivers tested with $14(87.5 \%)$ of 16 samples positive. Direct cultures that were negative were also negative by enrichment culture. Counts ranged from 1 to $5-\mathrm{cfu} / 100-\mathrm{ml}$. Seven $(43.7 \%)$ of 15 sea water samples from six sites on the Bristol Channel coastline of South Wales were positive with counts ranging from 3 to $6-\mathrm{cfu} / 100-\mathrm{ml}$, as were $7(46.7 \%)$ of 15 samples of lake water with counts ranging from 1 to 5-cfu/100-ml and $7(27 \%)$ of 26 samples of inland drainage. Of the treated waters, 4 of 8 swimming pool and 1 of 18 tap water $(5.5 \%)$ were positive with counts ranging from 1 to $3-\mathrm{cfu} / 100-\mathrm{ml}$. Overall, $84.6 \%$ of isolates from water samples produced toxin $\mathrm{A}$.

\section{Faecal specimens}

C. difficile was isolated from 10 of 100 samples of $\mathrm{dog}$ faeces and from two of 100 samples of cat faeces. Of these, two of 10 and one of two were positive for toxin A, respectively. Of 524 samples of faeces from an assortment of farm animals, the results by animal type were as follows: cattle none of 100 , sheep one of 100 , pigs none of 100 , poultry two of 120 , horse one of 100 . C. difficile was not isolated from 107 swabs of the gut contents of an assortment of fresh and sea water fish.
Table 1. Summary results for $C$. difficile in all samples

\begin{tabular}{lccc}
\hline Source & $\mathrm{n}$ & $\begin{array}{c}\text { Number (\%) } \\
\text { samples } \\
\text { positive }\end{array}$ & $\begin{array}{c}\text { Number (\%) } \\
\text { isolates } \\
\text { toxin A positive }\end{array}$ \\
\hline Dogs & 100 & $10(10)$ & $2(20)$ \\
Cats & 100 & $2(2)$ & $1(50)$ \\
Horses & 100 & $1(1)$ & $1(100)$ \\
Cattle & 104 & 0 & 0 \\
Sheep & 100 & $1(1)$ & $1(100)$ \\
Pigs & 100 & 0 & 0 \\
Fish & 107 & 0 & 0 \\
Poultry & 120 & $2(1.6)$ & $2(100)$ \\
Soil & 104 & $22(21.4)$ & $9(40.9)$ \\
Hospitals & 380 & $76(20)$ & $72(94.7)$ \\
Nursing homes & 275 & $6(2.2)$ & $4(66.7)$ \\
Family houses & 350 & $8(2.3)$ & $3(37.5)$ \\
Student residences & 200 & $4(2)$ & $3(75)$ \\
Veterinary clinics & 30 & $5(16.7)$ & $1(20)$ \\
Water samples & 110 & $40(36)$ & $36(90)$ \\
Vegetables & 300 & $7(2.3)$ & $5(71.4)$ \\
& & & \\
Total & 2580 & $184(7.1)$ & $140(76)$ \\
\hline
\end{tabular}

\section{Soil}

C. difficile was isolated from 22 (21\%) 104 of soil samples taken from public parks, gardens, playgrounds, etc. in the suburbs of Cardiff. Less than half these isolates $(40.9 \%)$ produced toxin A.

\section{Environmental surface samples}

A total of 1235 environmental samples was examined from hospital wards, veterinary clinics, nursing homes and private residences. Of these, $20 \%$ of 380 samples

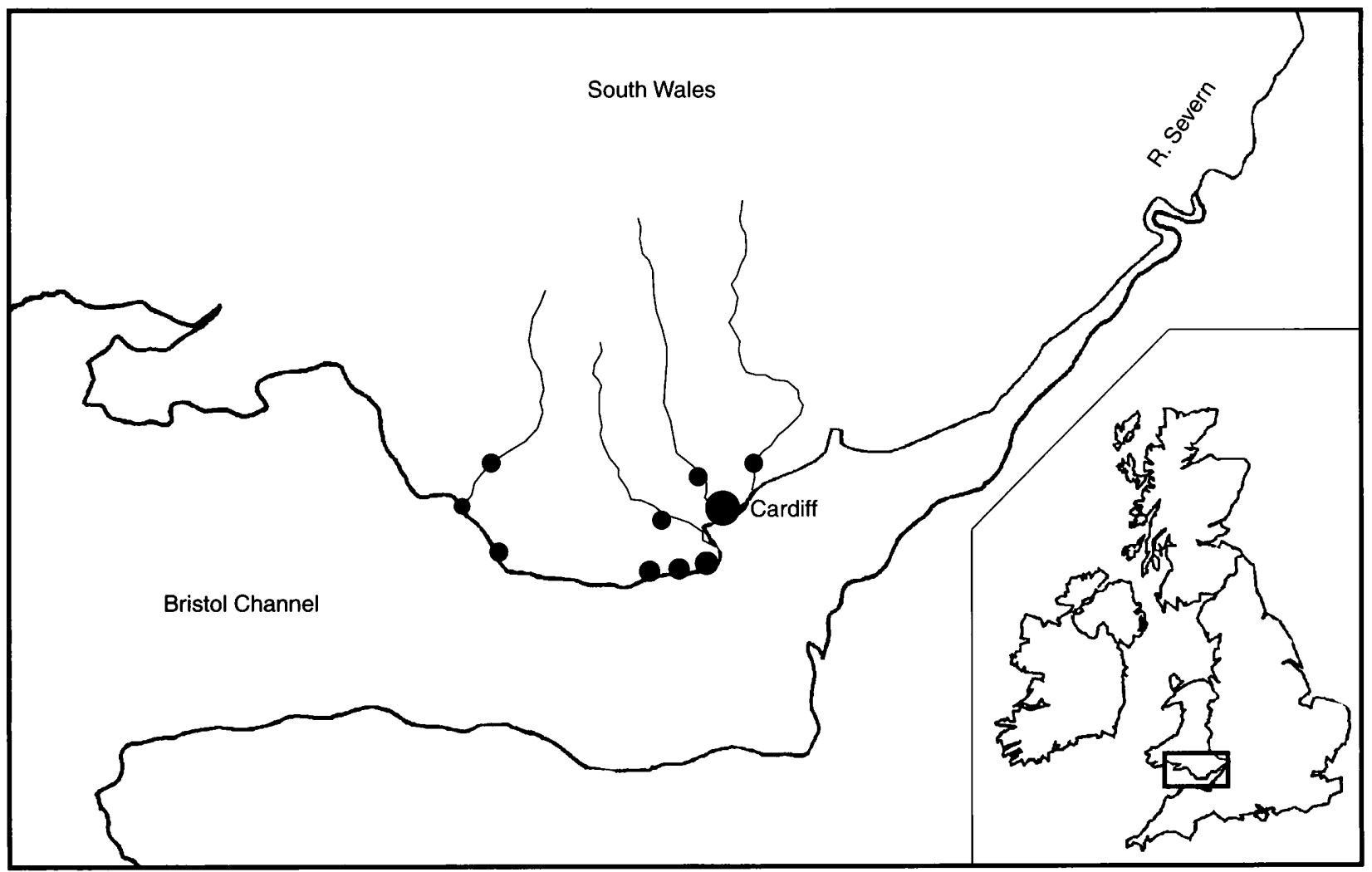

Fig. 1. Sampling sites of river and sea water for $C$. difficile. 
from hospitals were positive for $C$. difficile, as were $16.7 \%$ of 30 samples from the veterinary clinics, $2.6 \%$ of 275 samples from nursing homes and $2.2 \%$ of 550 samples from private residences. Nearly all $(94.7 \%)$ of the isolates from hospitals produced toxin A, compared with $37.5 \%$ and $47 \%$ of isolates from private residences and nursing homes, respectively.

\section{Raw vegetables}

Three hundred cultures taken from assorted raw vegetables yielded seven isolates of $C$. difficile. These isolates were from potato (two), onion (one), mushroom (one), carrot (one), radish (one) and cucumber (one). Five of seven isolates produced toxin A.

\section{Discussion}

The purpose of this study was to determine the prevalence of $C$. difficile in foodstuffs, water and the general environment in an attempt to demonstrate how the population at large may be exposed to this organism during normal everyday life. As a spore former, $C$. difficile can theoretically survive indefinitely in the environment and could, therefore, be ingested by man via various routes. For example, if it were shown to be a common member of the faecal flora of a livestock animal, it could contaminate meat at slaughter; or, if it was widely distributed in soil it could contaminate raw food such as fresh vegetables. Despite isolated reports of $C$. difficile in two sick piglets [13] and in one of 162 Nigerian cattle [14], there has not been a large survey of the prevalence of C. difficile in the gut of animals in the human food chain. The results of the present study suggest that consumption of beef, pork or lamb is not likely to be an important source of exposure to $C$. difficile, as it was not part of the faecal flora of 104 cattle, 100 pigs and $1 \%$ of sheep. Likewise, with only $1.6 \%$ of poultry faeces containing $C$. difficile, this food source also appears to have a low risk potential. The absence of $C$. difficile from the gut contents of fish, despite the organism being present in sea water, is in agreement with other studies of the clostridial flora of fish and fish products in which $C$. difficile has not been reported [15-16]. All these data suggest that $C$. difficile does not readily colonise the normal gut of farm animals or fish, probably due to competitive inhibition of $C$. difficile by the resident microbiota.

In addition to the potential contamination of the human food chain, it follows also that the urban population could be exposed to $C$. difficile via water if the spores of the organism were not removed by the sewage and water treatment system. The samples of potable water that were examined in the present study were included to test this theory, and although only one sample of chlorinated tap water was positive, the high percentage of river $(81.2 \%)$ and lake water $(40 \%)$ samples positive for $C$. difficile demonstrate the potential of water supplies as a source of infection should treatment fail. Although the presence of other clostridial species in water supplies has been demonstrated in several studies $[17,18], C$. difficile has not been reported. To our knowledge, the present study is the first to report the presence of $C$. difficile in untreated fresh water, sea water and chlorinated water, and represents a significant contribution to our understanding of the ecology of this organism.

C. difficile has been found in the sediments beneath marine and fresh water, however, and two isolates were reported in the sea water in Puget Sound off Washington State, USA [19]. Willis [17] sampled 43 waters from consumer premises in the Leeds area of northern England and found $88 \%$ to contain various clostridia in numbers ranging from 2 to $30 / 40-\mathrm{ml}$ of water. He found some seasonal fluctuations as the bacterial counts were generally higher in the colder months than in the summer. This was believed to be due to the algal/zoogleal layer in the sand filtration beds being less developed in the winter months, hence the filtration of micro-organisms by the sand beds is less efficient. It is of interest to note, therefore, that our sampling too was carried out over the autumn and winter months. The presence of $C$. perfringens in a treated water supply is thought to indicate a failure in the filtration process, and Nankivell [20] proposed that the sand beds themselves were a reservoir of clostridia, a theory that Willis [17] validated by isolating 'anaerobic bacilli' from 50 (91\%) of 55 sand filters. One of the rivers sampled in the present study had a sewage treatment plant sited on its western bank c. 0.5 mile from the coast. Two samples of river water taken upstream from this treatment plant were positive for $C$. difficile and two samples downstream from the plant were also positive, indicating contamination above the outlet of the treatment plant. The high percentage $(84.6 \%)$ of toxigenic isolates found in water is of interest, and typing studies are needed to establish the relationship between these strains and those from hospitals. The presence of $C$. difficile in swimming pool water is a finding that perhaps could be explained by a carrier, such as a young baby, using the pool. At this stage it is not possible to determine if the demonstration of an unexpectedly high prevalence of $C$. difficile in water is peculiar to the environment of South Wales, or due to the improved methodology used in this study. It was not surprising that samples from the Bristol Channel, into which the four culture-positive rivers flow, were also positive for $C$. difficile - although this finding does indicate that spores can survive the salinity of sea water.

Another potentially significant finding was the isolation of $C$. difficile from $21 \%$ of soil samples and 
$2.4 \%$ of unwashed raw vegetables on sale in retail outlets. To our knowledge this is the first report to demonstrate that root vegetables are a potential source of $C$. difficile infection in man.

The results from the environmental samples taken from hospital sites and veterinary clinics were not unexpected, as nosocomial distribution of $C$. difficile has been well documented. Perhaps of more interest was the isolation of the organism from $c .2 \%$ of private residences, indicating that some people are exposed to $C$. difficile in their own home. The findings of $C$. difficile in the faeces of dogs and cats corroborates earlier reports [21,22], although the percentage of positive cultures in dogs $(10 \%)$ and cats $(2 \%)$ was lower than reported in some studies [23].

The proportionally high detection rates of $C$. difficile in this study compared to earlier environmental studies may be attributable to the large nature of this study plus the use of a medium with spore germinating properties with prolonged incubation times to maximise recovery. On several occasions cultures that were negative after incubation for $48 \mathrm{~h}$ became positive after $72-120 \mathrm{~h}$. Typing studies are needed to establish whether the strains of $C$. difficile in the environment are similar to those found in hospitals, as well as further sampling to determine whether the organism is equally abundant in other regions of Britain, particularly in water supplies, rivers and the sea.

We gratefully acknowledge the assistance of the Heath Veterinary Group and the Croeso Veterinary Hospital for providing the pet samples, Dr Folasade Ogunsola for help with sampling, Mrs Sue Burge for help with processing the water samples, Drs Quoraishi, Hosein and Morgan for arranging the hospital sampling in Cardiff, and the individual farmers, shopkeepers and home owners for permission to sample on their property.

\section{References}

1. George RH, Symonds JM, Dimock F et al. Identification of Clostridium difficile as a cause of pseudomembranous colitis. BMJ 1978; 1: 695.

2. George WL, Sutter VL, Finegold SM. Antimicrobial agent- induced diarrhea - a bacterial disease. J Infect Dis 1977; 136: $822-828$.

3. Mulligan ME, Rolfe RD, Finegold SM, George WL. Contamination of a hospital environment by Clostridium difficile. Curr Microbiol 1979; 3: 173-175.

4. Kim KH, Fekety R, Batts DH et al. Isolation of Clostridium difficile from the environment and contacts of patients with antibiotic-associated colitis. $J$ Infect Dis 1981; 143: 42-50.

5. McFarland LV, Mulligan ME, Kwok RYY, Stamm WE, Nosocomial acquisition of Clostridium difficile infection. $N$ Engl J Med 1989; 320: 204-210.

6. Hafiz S, Oakley $\mathrm{CL}$. Clostridium difficile: isolation and characteristics. J Med Microbiol 1976; 9: 129-136.

7. Lindberg RB, Wetzler TF, Marshall JD, Newton A, Strawitz JG, Howard JM. The bacterial flora of battle wounds at the time of debridement. A study of the Korean battle casualty. Ann Surg 1955; 141: 369-374.

8. Blawat F, Chylinski G. Pathogenic clostridia in soil and faeces of domestic animals in the Gdansk region. Bull Inst Marine Med (Gdansk) 1958; 9: 117-126

9. Riley TV. The epidemiology of Clostridium difficile-associated diarrhoea. Rev Med Microbiol 1994, 5: 117-122.

10. Riley TV, Wetherall F, Bowman J, Mogyorosy J, Golledge CL. Diarrhoeal disease due to Clostridium difficile in general practice. Pathology 1991; 23: 346-349.

11. Brazier JS. Role of the laboratory in investigations of Clostridium difficile diarrhea. Clin Infect Dis 1993; 16 Suppl 4: S228-S233.

12. Borriello SP, Honour P. Simplified procedure for the routine isolation of Clostridium difficile from faeces. J Clin Pathol 1981; 34: 1124-1127.

13. Jones MA, Hunter D. Isolation of Clostridium difficile from pigs. Vet Rec 1983; 112: 253.

14. Princewell TJT, Agba MI. Examination of bovine faeces for the isolation and identification of Clostridium species. $J \mathrm{Appl}$ Bacteriol 1982; 52: 97-102.

15. Sakata T, Sugita H, Misuoka T, Kakimoto D, Kadota H. Isolation and distribution of anaerobic bacteria from the intestines of freshwater fish. Bull Jap Soc Sci Fish 1980; 46: 1249-1255.

16. Dodds $\mathrm{KL}$, Brodsky $\mathrm{MH}$, Warburton DW. A retail survey of smoked ready to eat fish to determine their microbiological quality. J Food Protect 1992; 55: 208-210.

17. Willis AT. Anaerobic bacilli in a treated water supply. $J$ Appl Bacteriol 1957; 20: 61-64.

18. Davies JA. Isolation and identification of clostridia from North sea Sediments. J Appl Bacteriol 1969; 32: 164-169.

19. Matches JR, Liston J. Mesophilic clostridia in Puget sound. Can J Microbiol 1974; 20: 1-7.

20. Nankivell AT. The sand filtration and purification of chalk waters. $J$ Hyg 1911; 11: 235-258.

21. Borriello SP, Honour P, Turner T, Barclay F. Household pets as a potential reservoir for Clostridium difficile infection. $J$ Clin Pathol 1983; 36: 84-87.

22. Riley TV, Adams JE, O'Neill GL, Bowman RA. Gastrointestinal carriage of Clostridium difficile in cats and dogs attending veterinary clinics. Epidemiol Infect 1991; 107: 659-665.

23. Perrin J, Buogo C, Gallusser A, Burnens AP, Nicolet J. Intestinal carriage of Clostridium difficile in neonate dogs. Zentralbl Veterinarmed B 1993; 40: 222-226. 